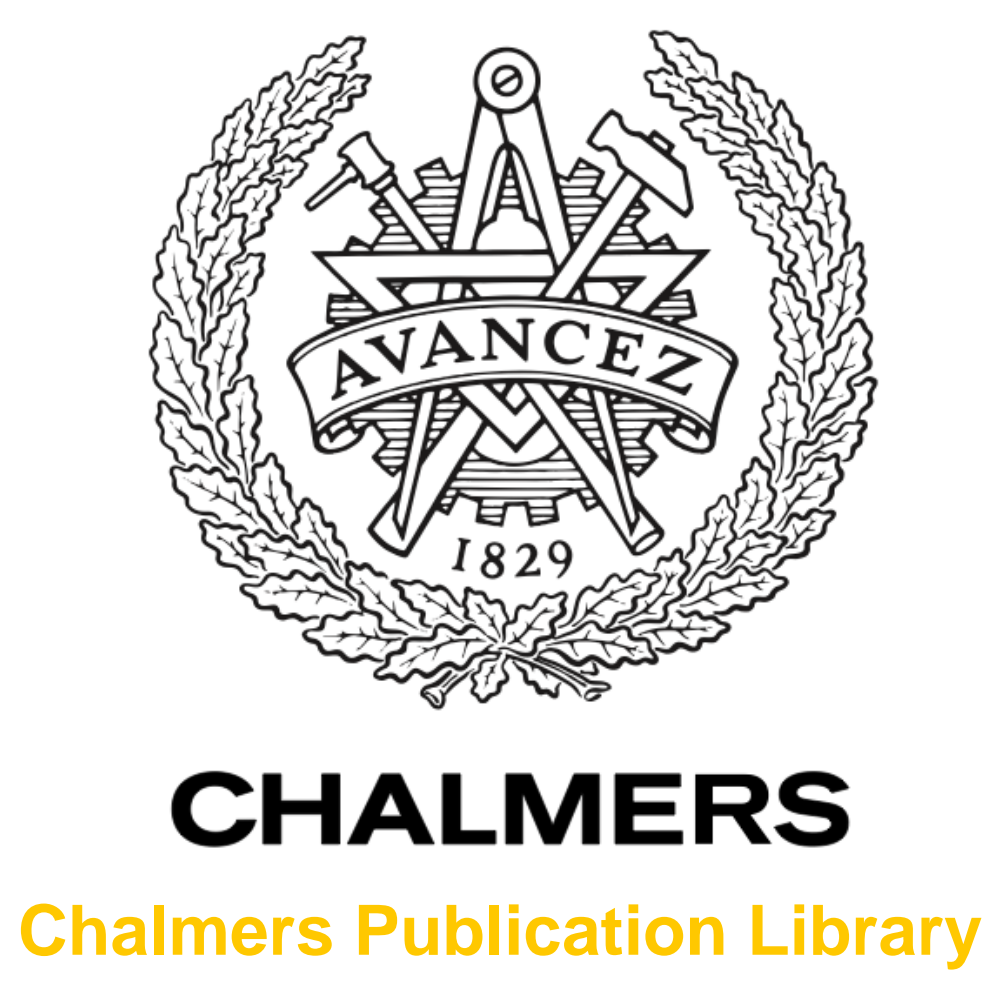

\title{
Comparison of Terminated and Tailbiting Spatially Coupled LDPC Codes With Optimized Bit Mapping for PM-64-QAM
}

This document has been downloaded from Chalmers Publication Library (CPL). It is the author's version of a work that was accepted for publication in:

2014 European Conference on Optical Communication, ECOC 2014; Cannes; France; 21 September 2014 through 25 September 2014

Citation for the published paper:

Häger, C. ; Graell i Amat, A. ; Brännström, F. (2014) "Comparison of Terminated and Tailbiting Spatially Coupled LDPC Codes With Optimized Bit Mapping for PM-64-QAM". 2014 European Conference on Optical Communication, ECOC 2014; Cannes; France; 21 September 2014 through 25 September 2014 pp. Art. no. 6964047.

http://dx.doi.org/10.1109/ECOC.2014.6964047

Downloaded from: http://publications.lib.chalmers.se/publication/199124

Notice: Changes introduced as a result of publishing processes such as copy-editing and formatting may not be reflected in this document. For a definitive version of this work, please refer to the published source. Please note that access to the published version might require a subscription. 


\title{
Comparison of Terminated and Tailbiting Spatially Coupled LDPC Codes With Optimized Bit Mapping for PM-64-QAM
}

\author{
Christian Häger ${ }^{(1)}$, Alexandre Graell i Amat ${ }^{(1)}$, Fredrik Brännström ${ }^{(1)}$, Alex Alvarado ${ }^{(2)}$, and Erik Agrell ${ }^{(1)}$ \\ (1) Department of Signals and Systems, Chalmers University, Sweden, 凶 christian.haeger@chalmers.se \\ (2) Department of Engineering, University of Cambridge, UK
}

\begin{abstract}
We optimize the allocation of coded bits to modulation bits for spatially coupled low-density parity-check (SC-LDPC) codes and PM-64-QAM. Tailbiting SC-LDPC codes are shown to offer a similar gap to capacity as their terminated counterparts, at a lower FEC overhead.
\end{abstract}

\section{Introduction}

There is currently a large interest in developing practical coded modulation (CM) schemes that can achieve high spectral efficiency close to the capacity limits of optical fibers ${ }^{1}$. Recently, SC-LDPC codes have attracted a lot of attention due to their capacity-achieving performance under belief propagation (BP) decoding for many communication channels ${ }^{2}$. They are considered as viable candidates for future spectrally efficient fiber-optical systems ${ }^{3-5}$.

The outstanding performance of SC-LDPC codes is due to a termination boundary effect which initiates a wave-like decoding behavior ${ }^{2}$. This behavior of terminated SC-LDPC codes comes at the price of a rate loss, i.e., a larger FEC overhead, compared to regular LDPC block codes. So-called tailbiting SC-LDPC codes may provide an interesting solution to this problem, since they do not suffer from an increased overhead. However, by default, tailbiting SC-LDPC codes behave essentially the same as regular LDPC block codes due to the absence of a termination boundary. In this paper, we demonstrate that the unequal error protection offered by the different modulation bits of a nonbinary modulation format can be exploited to create an artificial termination boundary. This significantly improves the performance of tailbiting SC-LDPC codes.

\section{System Model}

A block diagram of the considered polarizationmultiplexed (PM) fiber-optical transmission system is shown in Fig. 1. At each time instant $k$, the modulator $\Phi$ takes $m$ bits $b_{i, k}$ and maps them to a symbol $\mathbf{s}_{k}=\left(s_{\mathrm{x}, k}, s_{\mathrm{y}, k}\right)^{\top}$ taken from a discrete signal constellation $\mathcal{X} \subset \mathbb{C}^{2}$. We employ Gray-labeled PM-64-QAM as shown in Fig. 2 for each dimension, with $m=12$. The modulo 2 addition of the independent and uniformly distributed bits $d_{i, k}$ (and the multiplication by $\bar{d}_{i, k}=(-1)^{d_{i, k}}$ at the receiver) shown in Fig. 1 serves as a symmetrization technique ${ }^{6}$.

The baseband signal in each polarization is generated via a linear pulse modulation $s_{\mathrm{x}}(t)=\sum_{k} s_{\mathrm{x}, k} p(t-$ $k / R_{s}$ ) with pulse shape $p(t)$ and symbol rate $R_{s}$. The PM signal $\mathbf{s}(t)=\left(s_{\mathrm{x}}(t), s_{\mathrm{y}}(t)\right)^{\top}$ is launched into the fiber and propagates according to the Manakov equation $^{7}$. The optical link consists of $N_{\mathrm{sp}}$ spans of standard single-mode fiber (SSMF) with length $L_{\mathrm{sp}}$ and a lumped amplification scheme. Each erbium-doped fiber amplifier (EDFA) introduces circularly symmetric complex Gaussian noise with two-sided power spectral density (PSD) $N_{\mathrm{EDFA}}=\left(e^{\alpha L_{\mathrm{sp}}}-1\right) h \nu_{s} n_{\mathrm{sp}}$ per polarization ${ }^{1}$, where $\alpha$ is the attenuation coefficient, $h$ is Planck's constant, $\nu_{s}$ the carrier frequency, and $n_{\mathrm{sp}}$ the spontaneous emission factor. A standard coherent linear receiver according to $r_{\mathrm{x}, k}=\left.r_{\mathrm{x}}(t) * h(t) * p(-t)\right|_{t=k / R_{s}}$ is used, where $*$ denotes convolution and the frequency response of the equalizer $h(t)$ is $H(f)=$ $\exp \left(j 2 \beta_{2} \pi^{2} f^{2} N_{\mathrm{sp}} L_{\mathrm{sp}}\right)$. Based on the received samples $\mathbf{r}_{k}=\left(r_{\mathrm{x}, k}, r_{\mathrm{y}, k}\right)^{\top}$, the demodulator $\Phi^{-1}$ computes soft reliability information about the bits $b_{i, k}$ in the form of log-likelihood ratios (LLRs) $l_{i, k}$.

We consider a CM system according to the bitinterleaved coded modulation (BICM) paradigm, where a single binary code $\mathcal{C} \subset\{0,1\}^{n}$ of length $n$ and dimension $d$ is employed and each codeword $\mathbf{c}=\left(c_{1}, \ldots, c_{n}\right)$ is transmitted using $N=n / m$ symbols $\mathbf{s}_{k}$. The allocation of the coded bits to the modulation bits is determined by a bit mapper as shown in Fig. 3, where $\mathbf{u}=\left(u_{1}, \ldots, u_{d}\right)$ are the information bits.

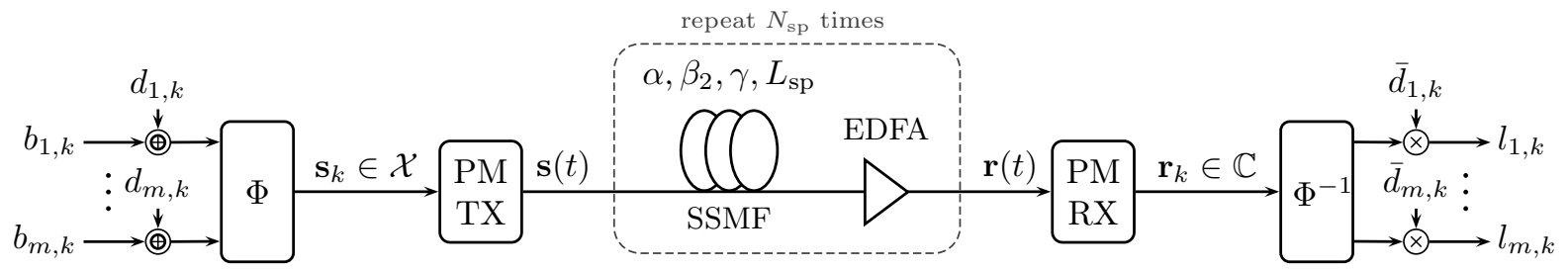

Fig. 1: Block diagram of the considered PM transmission system. 


\section{$\begin{array}{llllllll}000 & 001 & 011 & 010 & 110 & 111 & 101 & 100\end{array}$}

Fig. 2: Modulation bits per dimension.

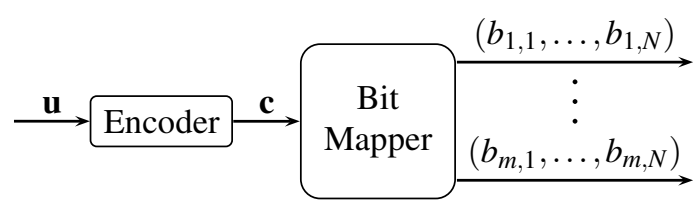

Fig. 3: Illustration of the Bit Mapper.

\section{Protograph-Based SC-LDPC Codes}

SC-LDPC codes have parity-check matrices with a band-diagonal structure and can be constructed using protographs $^{8}$. A protograph is a bipartite graph defined by a base matrix $\mathbf{P}=\left[p_{i, j}\right] \in \mathbb{N}_{0}^{c^{\prime} \times n^{\prime}}$. Given $\mathbf{P}$, the parity-check matrix $\mathbf{H}=\left[h_{i, j}\right] \in\{0,1\}^{c \times n}$ and corresponding Tanner graph of an LDPC code are obtained by replacing each entry $p_{i, j}$ in $\mathbf{P}$ with a random binary $M$-by- $M$ matrix with $p_{i, j}$ ones in each row and column, where $M$ is the lifting factor. The base matrix $\mathbf{P}_{[T]}$ of a $(J, K)$ regular SC-LDPC code with spatial length $T$ is constructed by specifying matrices $\mathbf{P}_{i}, 0 \leq i \leq m_{\mathrm{s}}$ of dimension $J^{\prime}$ by $K^{\prime}$. The matrices are such that $\mathbf{P}=\sum_{i=0}^{m_{\mathrm{s}}} \mathbf{P}_{i}$ has column weight $J$ and row weight $K$ for all columns and rows. Given the matrices $\mathbf{P}_{i}$ and $T$, one can construct $\mathbf{P}_{[T]}$ as shown in Fig. 4 (a) for the terminated case and in (b) for the tailbiting case.

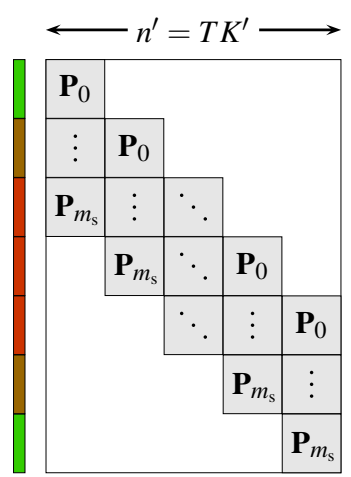

(a) terminated

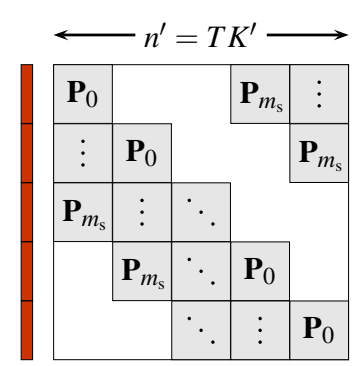

(b) tailbiting
Fig. 4: Base matrices for SC-LDPC codes.

By inspecting the strucutre of the base matrix for the terminated case, one may verify that the check node (CN) degrees corresponding to the first and last couple of rows is lower than the $\mathrm{CN}$ degrees corresponding to the rows in between. The lower degree $\mathrm{CNs}$ lead to a locally better decoding capability, which is visualized by the colored scale (green indicates a better correction capability), at the expense of a rate loss. This termination boundary effect initiates the wave-like decoding behavior that is characteristic for terminated SC-LDPC codes $^{2}$. On the other hand, for the tailbiting case, all CNs have the same degree $J$, hence no rate loss is incurred and all positions are protected equally. However, this also prevents the initiation of a decoding wave.
Fig. 5: Optimized allocation to the most protected modulation bit for each spatial position of the tailbiting SC-LDPC code (green $=100 \%$, red $=0 \%)$.

Terminated and tailbiting SC-LDPC have design rates $R(T)=1-\left(T+m_{\mathrm{s}}\right) J^{\prime} /\left(T K^{\prime}\right)$ and $R=1-J^{\prime} / K^{\prime}$, respectively $^{8}$. The rate loss for the terminated codes can be made arbitrary small by letting $T \rightarrow \infty$, but this also leads to very long block lengths (assuming a fixed lifting factor $M$ ).

\section{Bit Mapper Optimization}

The different modulation bits (see Fig. 2) have different protection levels, which can be taken advantage of by optimizing the bit mapper, i.e., the allocation of coded bits to the modulation bits. For protograph codes, each $\mathrm{VN}$ in the protograph represents $M$ variable nodes (VNs) in the lifted Tanner graph, i.e., $M$ coded bits. Consider as a baseline the widely used consecutive bit mapper according to $b_{i, k}=c_{(k-1) m+i}$ for $1 \leq i \leq m$, $1 \leq k \leq N$. If we assume for example that a given protograph is lifted with a lifting factor $M$ which is divisible by $m$, the baseline bit mapper allocates $M / m$ coded bits for each protograph VN to each modulation bit.

The bit mapper is modeled by specifying the assignment of protograph VNs to the modulation bits via a matrix $\mathbf{A}=\left[a_{i, j}\right] \in \mathbb{R}^{m \times n^{\prime}}$, where $a_{i, j}, 0 \leq a_{i, j} \leq 1$ $\forall i, j$ denotes the proportional allocation of the coded bits corresponding to the $j$ th column in the base matrix allocated to the $i$ th modulation bit. We optimize A based on a modified protograph extrinsic information transfer (P-EXIT) analysis. The P-EXIT analysis is used to predict the asymptotic decoding behavior assuming infinite codeword length for an additive white Gaussian noise (AWGN) channel. For more details about this procedure, we refer the reader to ${ }^{9}$. Once an optimized bit mapping matrix $\mathbf{A}^{*}$ is found, the finitelength bit mapper is obtained via the rounded matrix $M \mathrm{~A}^{*}$, from which the index assignment of coded bits to modulation bits is determined.

\section{Results and Discussion}

We consider $\mathbf{P}_{0}=(1,2,1,2)$ and $\mathbf{P}_{1}=(3,2,3,2)$ according to ${ }^{10}$ with $T=30$ and $M=3000$. The rates are given by $R(30) \approx 0.741$ and $R=0.75$ for the terminated and tailbiting codes, respectively. To reduce the decoding complexity and latency, we employ the windowed decoder (WD) developed in ${ }^{11}$ with a window size of $W=5$ and $l_{\max }=10$ iterations per window, which is taken into account in the bit mapper optimization. In Fig. 5, the optimized allocation of the coded bits of the tailbiting code to the most protected modulation bit is schematically shown. For the first spatial position (i.e., the first four columns in the base matrix), the coded bits are almost exclusively allocated to the best modulation bit, which leads to a locally improved decoding convergence and initiates a wave-like decoding 


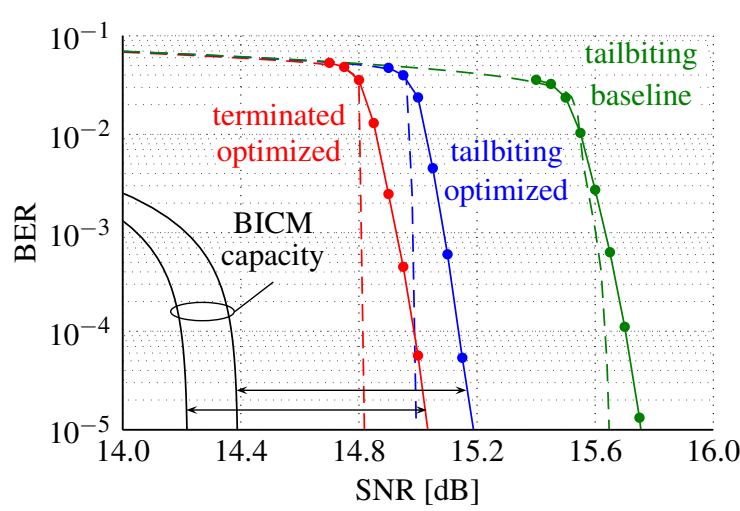

Fig. 6: Results for the AWGN channel.

behavior similar to that of terminated codes.

To compare the bit error rate (BER) performance of the terminated and tailbiting codes, in Fig. 6 we show simulation results (solid lines with markers) and the analytical P-EXIT prediction (dashed lines) for the AWGN channel. To allow for a fair comparison, we also optimized the bit mapper for the terminated code. The tailbiting code performs significantly better with an optimized bit mapper and a gain of $\approx 0.55 \mathrm{~dB}$ is achieved at a BER of $10^{-5}$. The terminated code performs better for the same signal-to-noise ratio (SNR), but entails a smaller spectral efficiency due to the rate loss. The gap to the BICM capacity of the two optimized systems, as indicated by the arrows, is approximately the same (as is the gap to the AWGN channel capacity, not shown).

Lastly, we present simulation results for the PM system assuming a single-channel transmission scenario including nonlinear effects. We set $\alpha=0.25 \mathrm{~dB} / \mathrm{km}$, $\beta_{2}=-21.668 \mathrm{ps}^{2} / \mathrm{km}, \gamma=1.4 \mathrm{~W}^{-1} \mathrm{~km}^{-1}, \nu_{s}=$ $1.934 \times 10^{14} \mathrm{~Hz}, n_{\mathrm{sp}}=1.622, R_{s}=40$ GBaud and $L_{\mathrm{sp}}=70 \mathrm{~km}$. A root-raised cosine pulse $p(t)$ with a roll-off factor of 0.25 is used. We employ the symmetric split-step Fourier method (SSFM) with two samples per symbol and a fixed step size of $\Delta=\left(10^{-4} L_{\mathrm{D}}^{2} L_{\mathrm{NL}}\right)^{1 / 3}$, where $L_{\mathrm{D}}=1 /\left(\left|\beta_{2}\right| R_{s}^{2}\right)$ and $L_{\mathrm{NL}}=1 /(\gamma P)$. The input power per polarization is fixed to $P=-2.5 \mathrm{dBm}$. In the simulation model, the polarization state is assumed to be known and perfect timing and carrier synchronization is assumed. In Fig. 7, the simulated BER of the PM transmission systems are plotted as a function of the number of fiber spans $N_{\mathrm{sp}}$. For the tailbiting code, the $0.55 \mathrm{~dB}$ gain obtained by using the optimized bit mapper for the AWGN channel translates into an increase of the transmission reach by roughly 3 additional spans or approximately $13 \%$. This gain is obtained at almost no increased system complexity cost, i.e., by replacing the baseline bit mapper with the optimized one. The terminated code enables a longer transmission reach of approximately one span, at the expense of a $1.2 \%$ decrease in spectral efficiency.

\section{Conclusion}

In this paper, we compared terminated and tailbiting SC-LDPC codes for a fiber-optical transmission system

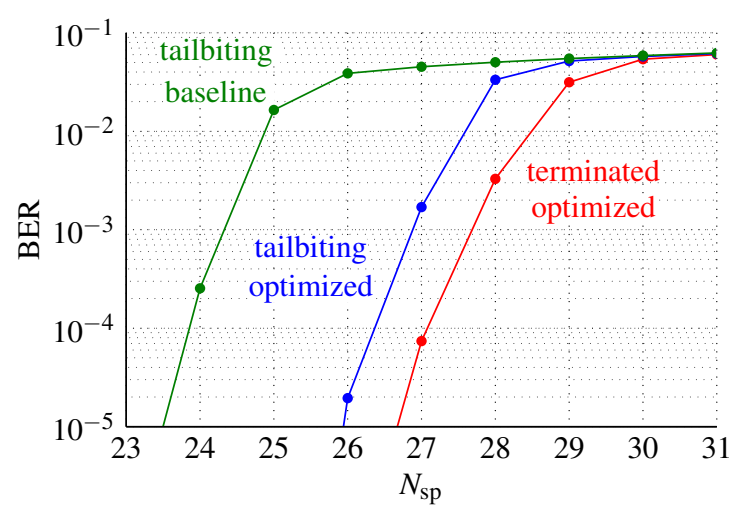

Fig. 7: Simulation results for the PM transmission system over a dispersion uncompensated transmission link.

employing PM-64-QAM. By optimizing the bit mapper for tailbiting SC-LDPC codes, a wave-like decoding behavior can be achieved which leads to a significantly improved performance. With an optimized bit mapper, the two codes are competitive, in the sense that spectral efficiency can be traded for transmission reach, at approximately the same gap to capacity.

\section{Acknowledgements}

This work was partially funded by the Swedish Research Council under grant \#2011-5961. The simulations were performed in part on resources provided by the Swedish National Infrastructure for Computing (SNIC) at C3SE.

\section{References}

[1] R.-J. Essiambre et al., "Capacity limits of optical fiber networks," J. Lightw. Technol., vol. 28, no. 4, p. 662 (2010).

[2] S. Kudekar et al., "Threshold saturation via spatial coupling: Why convolutional LDPC ensembles perform so well over the BEC," IEEE Trans. Inf. Theory, vol. 57, no. 2, p. 803 (2011).

[3] L. Schmalen et al., "Forward error correction in optical core and optical access networks," Bell Labs Tech. J, vol. 18 , no. 3, p. 39 (2013).

[4] A. Leven and L. Schmalen, "Status and recent advances on forward error correction technologies for lightwave systems," in Proc. ECOC, We.2.C.1, London (2013).

[5] Y. Miyata et al., "A spatially-coupled type LDPC code with an NCG of $12 \mathrm{~dB}$ for optical transmission beyond 100 Gb/s," in Proc. OFC, OM2B.4, Anaheim (2013).

[6] J. Hou et al., "Capacity-approaching bandwidth-efficient coded modulation schemes based on low-density paritycheck codes," IEEE Trans. Inf. Theory, vol. 49, no. 9, p. 2141 (2003).

[7] G. P. Agrawal, Lightwave Technology: Telecommunication Systems. Wiley-Interscience, Ch. 3 (2005).

[8] D. G. M. Mitchell et al., "AWGN channel analysis of terminated LDPC convolutional codes," Proc. ITA, La Jolla (2011).

[9] C. Häger et al., "Improving soft FEC performance for higher-order modulations by bit mapper optimization," arxiv:1404.2269 [cs.IT] (2014).

[10] L. Schmalen and S. ten Brink, "Combining spatially coupled LDPC codes with modulation and detection," in Proc. SCC, Munich (2013).

[11] A. R. lyengar et al., "Windowed decoding of protographbased LDPC convolutional codes over erasure channels," IEEE Trans. Inf. Theory, vol. 58, no. 4, p. 2303 (2012). 